\title{
The origin of cortical neurons
}

J.G. Parnavelas
Department of Anatomy and Developmental Biology, University College London, London, UK

\author{
Correspondence \\ J.G. Parnavelas \\ Department of Anatomy and \\ Developmental Biology \\ University College London \\ Gower Street \\ London WC1E 6BT \\ UK \\ Fax: +44-20-7679-7349 \\ E-mail: j.parnavelas@ucl.ac.uk \\ Presented at the IV International \\ UNESCO Course on "What the \\ Developing Cerebral Cortex Tells \\ About the Adult Cortex (and Vice \\ Versa)", Rio de Janeiro, RJ, Brazil, \\ December 3-7, 2001. \\ $\ldots \ldots \ldots \ldots \ldots \ldots$
}

Received July 12, 2002

Accepted October 16, 2002 $\ldots \ldots \ldots \ldots \ldots \ldots \ldots$

\begin{abstract}
Neurons of the mammalian cerebral cortex comprise two broad classes: pyramidal neurons, which project to distant targets, and the inhibitory nonpyramidal cells, the cortical interneurons. Pyramidal neurons are generated in the germinal ventricular zone, which lines the lateral ventricles, and migrate along the processes of radial glial cells to their positions in the developing cortex in an 'inside-out' sequence. The GABA-containing nonpyramidal cells originate for the most part in the ganglionic eminence, the primordium of the basal ganglia in the ventral telencephalon. These cells follow tangential migratory routes to enter the cortex and are in close association with the corticofugal axonal system. Once they enter the cortex, they move towards the ventricular zone, possibly to obtain positional information, before they migrate radially in the direction of the pial surface to take up their positions in the developing cortex. The mechanisms that guide interneurons throughout these long and complex migratory routes are currently under investigation.
\end{abstract}

Key words

- Neurogenesis

- Migration

- Cortical development

\section{Introduction}

The cerebral cortex is by far the largest part of the brain of mammals containing about half of the total number of neurons. Microscopic examination of a coronal section through the cortex shows that the neuronal cell bodies are arranged in six layers. The layering is produced by variation in the types and density of cell bodies through the cortical depth. Each layer contains a complement of pyramidal, so-called for the shape of their somata, and nonpyramidal neurons. Pyramidal neurons, which make up approximately $75 \%$ of all neurons in the cortex, are the projection cells that send axons to other areas of the cortex and to distant parts of the brain. They utilize the excitatory amino acid glutamate as a neurotransmitter. Nonpyramidal cells are interneurons, that is, their connections are all made locally. There are many varieties of nonpyramidal neurons based on differences in size and shape of their dendrites and patterns of axonal branching. They all contain the inhibitory neurotransmitter GABA and also one or more neuropeptides and/or calcium-binding proteins (for a review, see Ref. 1).

The cerebral cortex develops from the rostral dorsal part of the neural tube named the telencephalic pallium. The wall of the pallium is initially formed of neuroepithelial germinal cells whose continued proliferation causes the outward bulging of the pallial walls to form the cerebral vesicles. Neuroepithelial cells close to the ventricular lining of the neural tube, the so-called ventricular zone (VZ), are destined to give rise to neurons and some of the glial cells of the cortex. The radial alignment of the neuroepithelial cells imposes a radial pattern on the histogenesis of the pallium. The conventional scheme of cortical formation, de- 
picted in Figure $1(2,3)$, shows that early postmitotic neurons migrate away from the VZ towards the surface of the cerebral vesicles to form the primordial plexiform layer or preplate (PP). The later-generated neurons migrate to form a layer within the PP, the socalled cortical plate $(\mathrm{CP})$, thus splitting it into a superficial marginal zone (MZ; layer I) and a deep subplate (SP). The neurons of the CP assemble into layers II-VI in an 'inside-out' sequence, that is, the deepest cellular layers are assembled first and those closest to the surface last. Both the MZ and SP contain the earliest-generated neurons of the cerebral cortex. Those in layer I differentiate as CajalRetzius cells and other types of neurons that have not yet been fully characterized $(4,5)$. The SP is separated from the VZ by the intermediate zone (IZ), a layer that will eventually contain the afferent and efferent axonal tracts of the cortex (future white matter). As the $\mathrm{CP}$ emerges, another layer of proliferating cells appears between the VZ and IZ, the so-called subventricular zone (SVZ). This germinal zone contains cells, produced in the VZ, that mainly give rise to glia (6-8). The SVZ expands greatly in late gestation and in early postnatal life as the VZ disappears. According to this conventional scheme of cortical development, radial glial cells serve as a scaffold to support and direct the migration of young neurons from their origin in the VZ to their positions in the $\mathrm{CP}(9)$. However, this view is now changing in the light of emerging evidence which shows that radial glia produce cortical neurons and glia, in addition to guiding migration (10-13).

\section{Development of neuronal lineages}

At what point do undifferentiated cells in the VZ commit themselves to become pyramidal or nonpyramidal neurons? Knowledge of the genealogical (lineage) relationships of cells in the cortex would facilitate our efforts to understand the mechanisms that underlie their developmental choices. The study of cell lineages in the cerebral cortex has become possible with the use of recombinant retroviruses $(14,15)$. The advantage of this technique is that, by infecting single progenitor cells with a virus, a marker gene is introduced into them that can be inherited undiluted by all their progeny.

Earlier lineage studies, using predominantly the BAG retrovirus, observed singlelabeled cells or discrete clusters of two or more neurons in the cerebral cortex following the placement of intraventricular injec-
Figure 1. Three panels illustrate the layers of the cerebral wall at different stages of development. $V Z$, ventricular zone; $P P$, preplate; IZ, intermediate zone; $M Z$, marginal zone; $\mathrm{CP}$, cortical plate; SP, subplate; SV, subventricular zone.

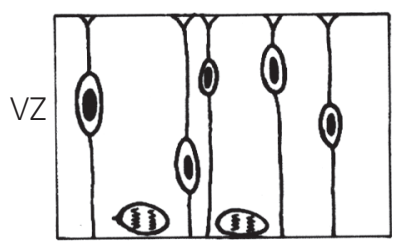

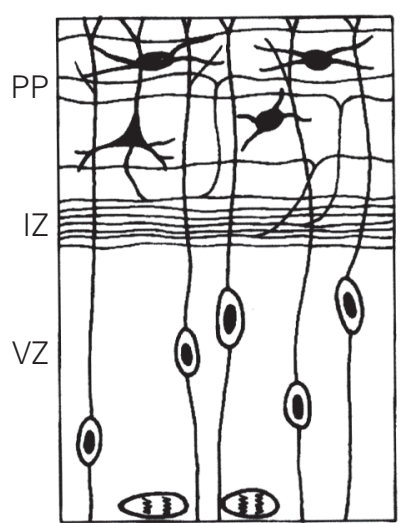

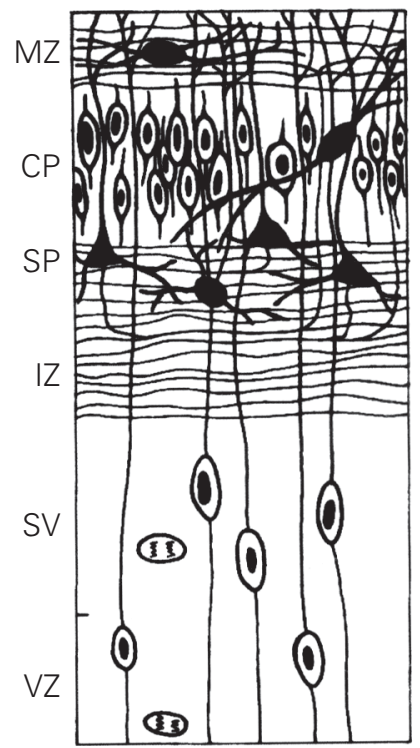


tions at any day of gestation during cortical neurogenesis in rats and mice $(16,17)$. The clones of two or more cells contained for the most part either neurons or glial cells, a finding that was confirmed in dissociated cell cultures $(16,18)$. When neuronal clones were examined further by electron microscopy or immunohistochemistry, it was found that they were nearly all homogeneous, composed of all pyramidal or all nonpyramidal cells (19-21). These early observations suggested that the VZ, the germinal layer of the embryonic cortex, might be viewed as a mosaic of progenitor cells each with a different restricted potential.

Further examination of clonally related neurons from brains injected with retrovirus at different stages of corticogenesis revealed remarkable differences in the number and laminar distribution of pyramidal and nonpyramidal neurons. Nonpyramidal cells appeared either as single cells or as clones of two neurons present in the same or in adjacent layers. Clones of pyramidal neurons were larger, and they were either dispersed in one layer or in several layers following earlier injections. Their size and laminar distribution was progressively reduced following later injections (20). This finding suggested the existence of different mechanisms that generate the two main neuronal types of the cerebral cortex. Other lineage analyses have utilized libraries of retroviral vectors carrying a large number of DNA tags, each of which can be distinguished by the polymerase chain reaction (22). These studies have shown that not all clonally related cells maintain the spatial relationship that they had before migration (23), but it is noteworthy that those that do invariably show the same phenotype (24).

The more recent use of a retroviral marker in combination with bromodeoxyuridine (BrdU) has shown that only pyramidal neurons maintain a close spatial relationship with their clonal relatives in the developing cortex, which can be achieved through radial migration (25). In contrast, labeled nonpyramidal cells were found, in agreement with earlier lineage analyses, as isolated cells or pairs of clonally neurons. Their low content of BrdU indicated that these cells were part of larger clones, and suggested that their isolation was the result of non-radial (tangential) migration. These findings pose two alternative interpretations: either clonally related cells, instructed to develop a particular phenotype, are also endowed with the ability to use a specific migratory pathway, or cues encountered during radial and tangential migration are responsible for the pyramidal and nonpyramidal phenotypes. Experiments by Tan et al. (26) have provided evidence for the former possibility with regards to the generation and migration of pyramidal neurons. Using highly unbalanced mouse embryonic stem cell chimeras, these authors found that radially dispersed neurons contained glutamate, the marker of pyramidal neurons, whereas tangentially dispersed cells were predominantly GABAergic. Their study has also demonstrated that specification of the pyramidal lineage occurs at the level of the progenitor, before the onset of neurogenesis.

Our understanding of the origin and development of nonpyramidal cell lineages is only now becoming clear. The studies of Mione et al. (25) have suggested that nonpyramidal cells, scattered in the cortex as isolated neurons or pairs of clonally related cells, were part of larger clones. This raised the question of whether there exist in the cortical VZ progenitors committed to producing only nonpyramidal cells. The analysis of chimeric mice by Tan and colleagues (26) raised the same question, and the authors concluded that whether these neurons are generated from progenitors with single or mixed potential, they have the tendency to be diffusely scattered in the cortex. We suggest that the dispersed sibling cells described in the lineage studies of Walsh and Cepko (23) were indeed nonpyramidal types, as 
were the neurons found to migrate tangentially in the developing cortex after they were labeled with a fluorescent marker in the VZ (27). The presence in the cortex of a relatively small number of nonpyramidal neurons compared with the often very large pyramidal clones suggests that there are other potential sources of nonpyramidal neurons.

\section{Nonpyramidal neurons: origin and tangential migration}

Sources of neurons destined for the cerebral cortex have been discovered in the ganglionic eminence, the primordium of the basal ganglia in the ventral telencephalon or subpallium. Porteus et al. (28) first reported that cells expressing $D l x 2$ appear to migrate from the ventral telencephalon to the neocortex. Subsequently, De Carlos et al. (29) and Tamamaki et al. (30) provided unequivocal evidence that cells in the lateral ganglionic eminence (LGE), the primordium of the striatum, migrate into the developing neocortex, and are distributed predominantly in the IZ. More recently, a number of tracing studies (31-33) have shown that the cells that
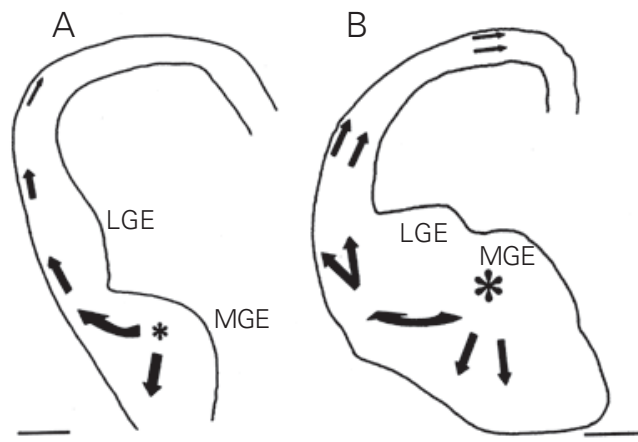

Figure 2. Camera lucida drawings of coronal sections through part of the rat forebrain after placement of DiI crystals in the medial ganglionic eminence (MGE) at E14 (A) and E16 (B). Arrows indicate the direction of migration of labeled cells in the cortical primordium 2 days (A) and 3 days (B) after DiI application. In $A$, fluorescent cells appeared as a stream rounding the corticostriatal sulcus and heading toward the preplate. In $B$, migrating cells were seen in the most superficial and the deeper aspects of the cortical primordium. LGE, lateral ganglionic eminence. migrate from the ventral telencephalon to the cortex are indeed GABAergic. Three different streams of neurons originating in the ventral telencephalon were observed to round the corticostriatal notch and follow tangentially oriented paths to enter the cortex. An early cohort (E12 in mouse; E14 in rat), originating in the medial ganglionic eminence (MGE), invades mainly the PP. These cells are tangentially oriented and show features typical of Cajal-Retzius cells (Figure $2 \mathrm{~A}$ ). A second and more prominent cohort, composed also of MGE cells, has been observed to migrate predominantly through the IZ slightly later in development (E13-15 mouse; E15-17 rat) (Figure 2B). At the late stages of corticogenesis, cells originating in the LGE and MGE appear in the lower IZ and SVZ (34). Genetic manipulations have provided further evidence for the origin of the cortical GABAergic interneurons in the subpallium. Thus, mice lacking transcription factors that regulate regionalization (Nkx2.1) or differentiation (Dlx1/2,Mash1) in the ventral telencephalon have significantly reduced numbers of GABAergic neurons in the cortex $(31,35,36)$. Specifically, $N k x 2.1$ mutants that lack a functional MGE show approximately a $50 \%$ reduction in GABA-containing neurons in the cortex, but the olfactory bulbs of these mutants contain nearly a normal number of GABAergic cells. Further, tracing studies in slice cultures of these mutants showed a significantly reduced migration of labeled cells in the cortex from the LGE or from the abnormal 'MGE'. In contrast, the $D l x 1 / 2$ mutants that lack migration of cells out of the LGE and MGE in slice cultures, have about a $75 \%$ reduction of GABA cells in the cortex and virtually no GABAergic cells in the olfactory bulb. These observations suggest that the MGE is the main source of interneurons to the cerebral cortex, while the LGE provides some cells to the cortex and many to the olfactory bulb. This is supported by the finding that dissociated MGE cells injected into the lateral ven- 
tricle tend to migrate to the striatum, pallidum and neocortex, while LGE cells migrate predominantly into the striatum and olfactory bulb (33). In a more recent study, Stühmer et al. (37) have shown that all cortical GABAergic neurons in the adult mouse brain are derived from cells that express the Dlx genes. This finding suggests that the vast majority, if not all, GABA-containing neurons in the mammalian cortex are derived from progenitors in the ventral telencephalon.

Neurons of the ganglionic eminence destined for the developing cerebral cortex express the LIM homeobox gene $\operatorname{Lhx} 6(32,38)$. In the later stages of corticogenesis and in postnatal life, Lhx6 has been found to be expressed in cells scattered throughout the cortical thickness. Although double-labeling experiments have not yet been conducted, the distribution of these cells resembles that of the GABA-containing neurons (Alifragis $P$ and Parnavelas JG, unpublished observations), suggesting that cortical interneurons express Lhx6 during corticogenesis and in later life. LIM homeodomain transcription factors have been previously implicated in neurotransmitter expression (39), and it may be that the expression of GABA in cortical interneurons is under the control of Lhx6.

The molecular mechanisms that guide the migration of interneurons from the ganglionic eminence, around the corticostriatal notch and into the neocortex are unknown. What signals trigger the migration of these neurons? A number of factors have been shown to stimulate motogenic activity in neural and non-neural tissues. One of these molecules, hepatocyte growth factor/scatter factor $(\mathrm{HGF} / \mathrm{SF})$, and its receptor MET have recently been shown to be important in the migration of cortical interneurons. Disruption of the normal expression of $\mathrm{HGF} / \mathrm{SF}$ appears to result in undirected scattering of cells from the ganglionic eminence and in a significant reduction of interneurons in the cortex at the time of birth (40). Recent studies have demonstrated that interneurons des- tined to populate the cortex express neuropilin1 and neuropilin2, which enable them to respond to chemorepulsion produced by class 3 semaphorins in the striatal mantle (41). Further, it appears that the repulsive activity of semaphorins in the developing striatum would create an exclusion zone for migrating cortical interneurons and channel them into adjacent paths, leading to the formation of MZ- and IZ-migratory routes.

Little is known about the substrates used by interneurons to reach the cortex, but their tangential migration appears largely independent of interactions with radial glia. However, a close association has been observed between tangentially oriented cells and corticofugal axons, predominantly in the IZ and $\mathrm{MZ}$ of the developing cortex (42), suggesting that interneurons may use this axonal system as a scaffold for their migration into the cortex. Earlier studies had suggested that axons can provide a substratum for nonradial migration in the developing CNS including the cerebral cortex $(43,44)$. Recent studies (45) have provided evidence that the neural adhesion molecule TAG-1, a member of the immunoglobulin superfamily present on corticofugal fibers, serves as a substrate upon which GABAergic interneurons migrate. Blocking TAG-1 function in cortical slices with anti-TAG-1 antibodies or soluble TAG-1 protein results in marked reduction of migrating GABAergic interneurons (45).

If tangentially migrating neurons use the corticofugal system to enter the neocortex, how do they integrate into specific layers with their pyramidal counterparts? Using time-lapse imaging of cortical slices together with tracer-labeling techniques, Nadarajah et al. (46) have shown that interneurons arising in the ventral telencephalon actively migrate towards the cortical VZ upon reaching the dorsal telencephalon, a mode of movement that has been termed 'ventricle-directed migration'. After a pause in this proliferative zone, they migrate radially in the direction of the pial surface to take up positions in the 
developing CP (Figure 3).

Earlier birthdating studies (47) and more recent transplantation studies (48) have demonstrated that, similar to pyramidal cells, cortical interneurons are disposed in an 'inside-out' pattern within the CP. How is it that two neuronal types generated in two distinct regions of the developing brain, the pallium and subpallium, and shown to follow different migratory paths, can assemble in the cortex in a way that they are linked temporally and spatially? Nadarajah and colleagues (46) have proposed that cortical in-
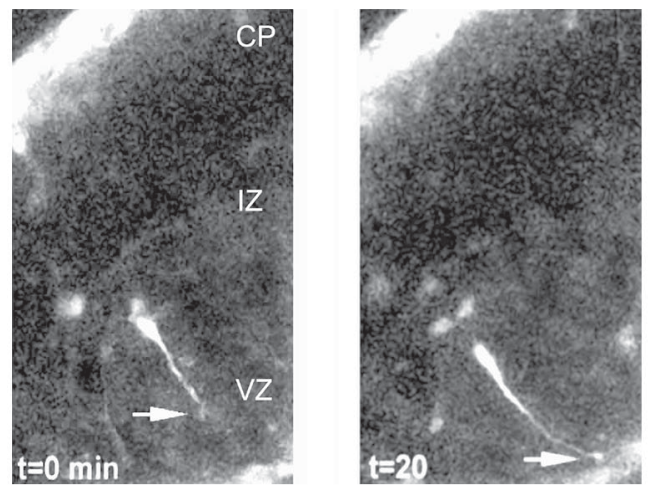

terneurons follow ventricle-directed migration actively seeking the VZ in order to obtain the type of positional information that the pyramidal cells acquire prior to becoming postmitotic. These cues may be obtained from the local environment or from the pyramidal cells themselves through neural-neural interactions. The mechanism that guides interneurons to the VZ before they move to the positions in the developing cortex is not known, but a combination of chemoattractant (e.g., semaphorins) and chemorepellent (e.g., Slit) molecules may be involved.
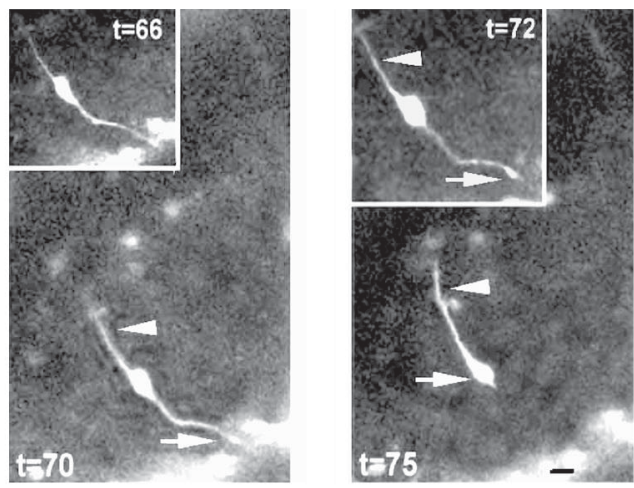

Figure 3. Time-lapse sequence of a cell undergoing ventricle-directed migration in an acute mouse cortical slice labeled with Oregon Green BAPTA-1 488 AM. The arrows point to the tip of the leading process of the cell as it rapidly advances towards the ventricular surface (bottom). The arrowheads point to a growing trailing process that will later become the leading process as the cell changes direction and moves towards the pial surface. Images were collected every minute and each frame shown here is a single optical section. CP, cortical plate; IZ, intermediate zone; VZ, ventricular zone; t, time. Scale bar, $20 \mu \mathrm{m}$.

\section{References}

1. Parnavelas JG, Dinopoulos A \& Davies SW (1989). The central visual pathways. In: Björklund A, Hökfelt T \& Swanson LW (Editors), Handbook of Chemical Neuroanatomy. Vol. 7. Integrated Systems of the CNS, Part II. Elsevier, Amsterdam, The Netherlands, 1-164.

2. Boulder Committee (1970). Embryonic vertebrate central nervous system: revised terminology. Anatomical Record, 166: 257-262.

3. Uylings HBM, Van Eden CG, Parnavelas JG \& Kalsbeek A (1990). The prenatal and postnatal development of rat cerebral cortex. In: Kolb B \& Tees RC (Editors), The Cerebral Cortex of the Rat. MIT Press, Cambridge, MA, USA, 35-76.

4. Frotscher M (1997). Dual role of Cajal-
Retzius cells and reelin in cortical development. Cell and Tissue Research, 290: 315-322.

5. Meyer G, Goffinet AM \& Fairén A (1999). What is a Cajal-Retzius cell? A reassessment of a classical cell type based on recent observations in the developing cortex. Cerebral Cortex, 9: 765-775.

6. Levison SW \& Goldman JE (1993). Both oligodendrocytes and astrocytes develop from progenitors in the subventricular zone of postnatal rat forebrain. Neuron, 10: 201-212

7. Luskin MB \& McDermott KA (1994). Divergent lineages for oligodendrocytes and astrocytes originating in the neonatal forebrain subventricular zone. Glia, 11: 211226.
8. Parnavelas JG (1999). Glial cell lineages in the rat cerebral cortex. Experimental Neurology, 156: 418-429.

9. Rakic P (1972). Mode of cell migration to the superficial layers of fetal monkey neocortex. Journal of Comparative Neurology 145: $61-83$

10. Hartfuss E, Galli R, Heins N \& Gotz M (2001). Characterization of CNS precursor subtypes and radial glia. Developmental Biology, 229: 15-30.

11. Miyata T, Kawaguchi A, Okano H \& Ogawa M (2001). Asymmetric inheritance of radial glial fibers by cortical neurons. Neuron, 31: 727-741.

12. Noctor SC, Flint AC, Weissman TA, Dammerman RS \& Kriegstein AR (2001). Neurons derived from radial glial cells estab- 
lish radial units in neocortex. Nature, 409: 714-720.

13. Parnavelas JG \& Nadarajah B (2001). Radial glial cells: are they really glia? Neuron, 31: 881-884.

14. Sanes JR, Rubenstein JLR \& Nicolas J-F (1986). Use of recombinant retrovirus to study post-implantation cell lineage in mouse embryos. EMBO Journal, 5: 31333142.

15. Price J, Turner D \& Cepko C (1987). Lineage analysis in the vertebrate nervous system by retrovirus-mediated gene transfer. Proceedings of the National Academy of Sciences, USA, 84: 156-160.

16. Luskin $M B$, Pearlman AL \& Sanes JR (1988). Cell lineage in the cerebral cortex of the mouse studied in vivo and in vitro with a recombinant retrovirus. Neuron, 1: 635-647.

17. Price J \& Thurlow L (1988). Cell lineage in the rat cerebral cortex: a study using retroviral-mediated gene transfer. Development, 104: 473-482.

18. Price J, Williams B \& Grove E (1992). The generation of cellular diversity in the cerebral cortex. Brain Pathology, 2: 23-29.

19. Parnavelas JG, Barfield JA, Franke E \& Luskin MB (1991). Separate progenitor cells give rise to pyramidal and nonpyramidal neurons in the rat telencephalon. Cerebral Cortex, 1: 463-468.

20. Luskin MB, Parnavelas JG \& Barfield JA (1993). Neurons, astrocytes, and oligodendrocytes of the rat cerebral cortex originate from separate progenitor cells: an ultrastructural analysis of clonally related cells. Journal of Neuroscience, 13: 1730-1750.

21. Mione MC, Danevic C, Boardman P, Harris B \& Parnavelas JG (1994). Lineage analysis reveals neurotransmitter (GABA and glutamate) but not calcium-binding protein homogeneity in clonally related cortical neurons. Journal of Neuroscience, 14 : 107-123.

22. Walsh C \& Cepko CL (1992). Widespread dispersion of neuronal clones across functional regions of the cerebral cortex. Science, 255: 434-440.

23. Walsh C \& Cepko CL (1993). Clonal dispersion in proliferative layers of developing cerebral cortex. Nature, 362: 632-635.

24. Reid CB, Liang I \& Walsh C (1995). Systematic widespread clonal organization in cerebral cortex. Neuron, 15: 299-310.

25. Mione MC, Cavanagh JFR, Harris B \& Parnavelas JG (1997). Cell fate specification and symmetrical/asymmetrical divisions in the developing cerebral cortex. Journal of Neuroscience, 17: 2018-2029.
26. Tan SS, Kalloniatis M, Sturm K, Tam PPL, Reese BE \& Faulkner-Jones B (1998). Separate progenitors for radial and tangential cell dispersion during development of the cerebral cortex. Neuron, 21: 295-304.

27. O'Rourke NA, Sullivan DP, Kaznowski CE, Jacobs AA \& McConnell SK (1995). Tangential migration of neurons in the developing cerebral cortex. Development, 121: 2165-2176

28. Porteus MH, Bulfone A, Liu JK, Puelles L, Lo LC \& Rubenstein JLR (1994). DLX-2, MASH-1, and MAP-2 expression and bromodeoxyuridine incorporation define molecularly distinct cell populations in the embryonic mouse forebrain. Journal of Neuroscience, 14: 6370-6383.

29. De Carlos JA, Lopez-Mascaraque L \& Valverde $F$ (1996). Dynamics of cell migration from the lateral ganglionic eminence in the rat. Journal of Neuroscience, 16: 6146-6156.

30. Tamamaki N, Fugimori KE \& Takauji R (1997). Origin and route of tangentially migrating neurons in the developing neocortical intermediate zone. Journal of Neuroscience, 17: 8313-8323.

31. Anderson SA, Eisenstat DD, Shi L \& Rubenstein JLR (1997). Interneuron migration from the basal forebrain to neocortex: dependence on Dlx genes. Science, 278: 474-476.

32. Lavdas AA, Grigoriou M, Pachnis V \& Parnavelas JG (1999). The medial ganglionic eminence gives rise to a population of early neurons in the developing cerebral cortex. Journal of Neuroscience, 19: 78817888.

33. Wichterle H, Garcia-Verdugo JM, Herrera DG \& Alvarez-Buylla A (1999). Young neurons from medial ganglionic eminence disperse in adult and embryonic brain. Nature Neuroscience, 2: 461-466.

34. Anderson SA, Marin O, Horn C, Jennings K \& Rubenstein JLR (2001). Distinct cortical migrations from the medial and lateral ganglionic eminences. Development, 128 : 353-363.

35. Casarosa S, Fode C \& Guillemot F (1999). Mash1 regulates neurogenesis in the ventral telencephalon. Development, 126: 525-534.

36. Sussel L, Marin O, Kimura S \& Rubenstein JLR (1999). Loss of Nkx2.1 homeobox gene function results in a ventral to dorsal molecular respecification within the basal telencephalon: evidence for a transformation of the pallidum into the striatum. Development, 126: 3359-3370.

37. Stühmer T, Puelles L, Ekker M \& Ruben- stein JLR (2002). Expression from a Dlx gene enhancer marks adult mouse cortical GABAergic neurons. Cerebral Cortex, 12: 75-85.

38. Grigoriou M, Tucker AS, Sharpe PT \& Pachnis V (1998). Expression and regulation of Lhx6 and Lhx7, a novel subfamily of LIM homeodomain encoding genes, suggests a role in mammalian head development. Development, 125: 2063-2074.

39. Thor S \& Thomas JB (1997). The Drosophila islet gene governs axon pathfinding and neurotransmitter identity. Neuron, 18 : 397-409.

40. Powell EM, Mars WM \& Levitt $P$ (2001). Hepatocyte growth factor/scatter factor is a motogen for interneurons migrating from the ventral to the dorsal telencephaIon. Neuron, 30: 79-89

41. Marin O, Yaron A, Bagri A, TessierLavigne M \& Rubenstein JL (2001). Sorting of striatal and cortical interneurons regulated by semaphorin-neuropilin interactions. Science, 293: 872-875.

42. Mètin C \& Godement $P$ (1996). The ganglionic eminence may be an intermediate target for corticofugal and thalamocortical axons. Journal of Neuroscience, 16: 32193235.

43. Rakic $P$ (1985). Contact regulation of neuronal migration. In: Edelman GM \& Thiery JP (Editors), The Cell in Contact. WileyLiss, Inc., New York, NY, USA, 67-91.

44. Gray GE, Leber SM \& Sanes JR (1990). Migratory patterns of clonally related cells in the developing central nervous system. Experientia, 46: 929-940.

45. Denaxa M, Chan C-H, Schachner M, Parnavelas JG \& Karagogeos D (2001). The adhesion molecule TAG-1 mediates the migration of cortical interneurons from the ganglionic eminence along the corticofugal fiber system. Development, 128: 46354644.

46. Nadarajah B, Alifragis P, Wong R \& Parnavelas JG (2002). Ventricle-directed migration in the developing cerebral cortex. $\mathrm{Na}$ ture Neuroscience, 5: 218-224.

47. Miller MW (1985). Cogeneration of retrogradely labeled corticocortical projection and GABA-immunoreactive local circuit neurons in cerebral cortex. Developmental Brain Research, 23: 187-192.

48. Wichterle H, Turnbull DH, Nery S, Fishell G \& Alvarez-Buylla A (2001). In utero fate mapping reveals distinct migratory pathways and fates of neurons born in the mammalian basal forebrain. Development, 128: 3759-3771. 